\title{
Regulation of trades based on differences in beliefs *
}

\author{
Hervé Crès ${ }^{\dagger} \quad$ Mich Tvede ${ }^{\ddagger}$
}

\begin{abstract}
Some trades based on differences in beliefs might cause more harm than good. Should they be restricted? If yes, how? We propose three properties ensuring that regulation does not prevent beneficial trade and is consistent: Unanimity - the regulator should not object to trades with identical beliefs; Merge-Proofness of Autarky - if the regulator does not object to finitely many unrelated trades, all with identical beliefs, then it should not object to the mere juxtaposition of the trades; and Independence of Irrelevant Trade - if the regulator does not object to the juxtaposition of two unrelated trades, then it should not object to any of the two trades standing alone. We show that there is a unique policy having these three properties, namely laissez-faire.
\end{abstract}

Keywords Heterogeneous Beliefs $\cdot$ Pareto Efficiency $\cdot$ Regulation $\cdot$ Speculative Trading JEL Classification D51 - D69

\footnotetext{
*We thank Darrell Duffie, Itzhak Gilboa, Larry Samuelson and Robert Sugden for insightful comments on earlier versions on this paper.

$\dagger$ New York University in Abu Dhabi, PO Box 129188 Abu Dhabi, United Arab Emirates, email: herve.cres@nyu.edu

†University of East Anglia, Norwich, NR4 7TJ, UK, email: m.tvede@uea.ac.uk
} 


\section{Introduction}

The sub-prime crisis has caused massive destruction of welfare reinforcing the suspicion that some trading activities on financial markets are potentially harmful. It was followed by a surge of scholarly interest in trying to identify and curb potentially harmful trades. Especially one category of trades has attracted lots of attention, namely trades based on differences in beliefs about the future.

Trades with heterogeneous beliefs are ex ante welfare improving for the agents involved. However different agents use different beliefs to evaluate trades. Hence the concern that agents make mistakes such that welfare gains turn out to be welfare losses. That is why some scholars consider Pareto domination to be an insufficient welfare criterion in case traders have heterogeneous beliefs. Several refinements of the Pareto domination have subsequently been proposed. These refinements aim at singling out, among all trades, those most likely to harm, and form the foundation for some tightening of the regulation of financial markets.

However, well-functioning financial markets create welfare by allowing agents to trade risks. Regulation should not prevent beneficial trades. Moreover for sets of fundamentals for which it is not obvious whether trades are beneficial or not the regulator should be able to argue for its decision by: pointing out its decision for other sets of fundamentals for which its decision is obvious; and explaining how the two sets of fundamentals are similar. In short, regulation should be consistent.

To meet these ends we propose three properties of regulation of trades with heterogeneous beliefs. First, the regulator should not object to trades with identical beliefs. Second, if the regulator does not object to finitely many unrelated trades, all with identical beliefs, then it should not object to the mere juxtaposition of the trades. Third, if the regulator does not object to a trade that is the juxtaposition of two trades for the juxtaposition of two autarkic economies, then it should not object to any of the sub-trades for the associated autarkic economy. We show that there is a unique policy having the three properties: Laissez-faire.

Obviously the result that solely laissez-faire has the three properties can be disturbing or soothing depending on personal conviction. But the result in itself should not be discussed because it is simply a logical consequence of the three properties. However the three properties can and should be discussed. Though we are biased in our evaluation of the three properties, we do provide a somewhat detailed discussion of them - both in the next subsection and in connection with the precise statements of them.

\section{Illustration}

To illustrate the problem and describe more precisely our contribution, let us introduce a series of examples. 
Example 1: (Gilboa et al., 2014) There are two risk neutral agents, Ann and Bill, both endowed with $\$ 1$. There are two states of the world: In the first state $s=1$ the oil price is above $\$ 100$ one year from now and in the second state $s=2$ it is below. Ann believes the probability of state $s=1$ is $2 / 3$. Bill believes the probability of state $s=1$ is $1 / 3$. Therefore their expected utility functions are

$$
U_{A}\left(x_{1}, x_{2}\right)=\frac{2}{3} x_{1}+\frac{1}{3} x_{2} \text { and } U_{B}\left(x_{1}, x_{2}\right)=\frac{1}{3} x_{1}+\frac{2}{3} x_{2} .
$$

They want to trade one for one such that they both receive $\$ 1$ in the state they deem more probable and deliver $\$ 1$ in the state they deem less probable. This trade increases their expected utilities from 1 to $4 / 3$.

In Example 1 the sole reason for Ann and Bill to trade is the difference in their beliefs. They have identical endowments and attitudes toward risk. Moreover they are risk-neutral and their endowments are state-independent, so they have no insurance needs. Ann and Bill are both taking advantage of the difference in their beliefs to speculate against each other, betting on the other having mistaken beliefs. It is a pure bet. The trade could be considered to cause more harm than good.

Some other trades would typically not be considered harmful, e.g., when for each agent resources flow from the state deemed more probable to the state deemed less probable. An example follows.

Example 2: There are the same two states as in Example 1. There are two agents with a constant relative risk aversion of one. Cai is endowed with $\$ 2$ in state $s=1$ and $\$ 0.1$ in state $s=2$, and believes the probability of state $s=1$ is $2 / 3$. Djal is endowed with $\$ 0.1$ in state $s=1$ and $\$ 2$ in state $s=2$, and believes the probability of state $s=1$ is $1 / 3$. Their expected utility functions are

$$
U_{C}\left(x_{1}, x_{2}\right)=\frac{2}{3} \ln \left(x_{1}\right)+\frac{1}{3} \ln \left(x_{2}\right) \text { and } U_{D}\left(x_{1}, x_{2}\right)=\frac{1}{3} \ln \left(x_{1}\right)+\frac{2}{3} \ln \left(x_{2}\right) .
$$

They want to trade one for one such that Djal receives $\$ 1$ in state $s=1$ and Cai receives $\$ 1$ in state $s=2$. This trade increases their expected utilities.

In Example 2 trading occurs between agents with different beliefs, but this is not crucial for justifying the trade. Indeed even if the two agents switched beliefs the trade would still increase their expected utilities. Consequently the agents do not seem to speculate against each other. Both agents are risk averse and the trade seems to be triggered by insurance needs stemming from large endowment shocks. This trade would typically be considered beneficial.

From a regulatory perspective it would be useful to separate potentially harmful, highly speculative trades, triggered by differences in beliefs (as in Example 1) from more beneficial 
trades, triggered by insurance needs (as in Example 2). To do this in a systematic way we adopt an axiomatic approach and identify three limits within which trades should not be banned in order to prevent excessive regulation.

\section{Three properties of regulation}

Let us proceed with more illustrating examples. Since the examples use the same four agents doing the same net-trades ending with the same final consumptions as in Examples 1 and 2, we summarize their characteristics and net-trades in Table 1.

\begin{tabular}{c|ccccc|} 
& Endowment & Belief & Elementary utility & Net-trade & Consumption \\
\hline Ann & $(1,1)$ & $\left(\frac{2}{3}, \frac{1}{3}\right)$ & $u(x)=x$ & $(1,-1)$ & $(2,0)$ \\
Bill & $(1,1)$ & $\left(\frac{1}{3}, \frac{2}{3}\right)$ & $u(x)=x$ & $(-1,1)$ & $(0,2)$ \\
Cai & $(2,0.1)$ & $\left(\frac{2}{3}, \frac{1}{3}\right)$ & $u(x)=\ln (x)$ & $(-1,1)$ \\
Djal & $(0.1,2)$ & $\left(\frac{1}{3}, \frac{2}{3}\right)$ & $u(x)=\ln (x)$ & $(1,-1)$
\end{tabular}

Table 1: Characteristics and trades.

Example 3: There are two agents: Ann from Example 1 and Cai from Example 2. They both believe that the probability of state $s=1$ is $2 / 3$. They want to trade one for one such that Ann receives $\$ 1$ in state $s=1$ and Cai receives $\$ 1$ in state $s=2$.

In Example 3 trading is based on insurance needs caused by differences in endowments and attitudes toward risk, but absolutely not on differences in beliefs: Ann and Cai have identical beliefs. Unless the regulator has additional information, the standard notion of Pareto domination should not be questioned. Hence we propose a first limit on regulation: If all agents involved in a trade have identical beliefs, then the trade should be authorized. We term this property: Unanimity (U).

The argument would be exactly the same in the following mirror image of Example 3.

Example 4: There are two agents: Bill from Example 1 and Djal from Example 2. They both believe that the probability of state $s=1$ is $1 / 3$. They want to trade one for one such that Djal receives $\$ 1$ in state $s=1$ and Bill receives $\$ 1$ in state $s=2$. 
To introduce our second limit on regulation, consider the juxtaposition of the two trades in Examples 3 and 4.

Example 5: There are four agents: Ann, Cai, Bill and Djal. Ann and Cai trade as described in Example 3 and Bill and Djal trade as described in Example 4.

There are agents with different beliefs in Example 5. Ann and Cai on one side believe the probability of state $s=1$ is $2 / 3$, and Bill and Djal on the other side believe the probability is $1 / 3$. However there is strictly no trade between agents with different beliefs, neither directly nor indirectly. Consequently trading is not in any sense based on differences in beliefs. The juxtaposition of the two trades does not introduce any speculation: Nobody is betting on others having mistaken beliefs. Therefore we propose a second limit on regulation: If $n$ trades, in $n$ autarkic economies, all involving agents with identical beliefs, are authorized, then the juxtaposed trade in the juxtaposed economy should be authorized. We term this property: Merge-Proofness of Autarky (MA).

To introduce our third limit on regulation, consider another way to frame Example 5.

Example 5': There are four agents: Ann, Bill, Cai and Djal. They start with initial allocation $((1,1),(1,1),(2,0.1),(0.1,2))$ and trade for final allocation $((2,0),(0,2),(1,1.1),(1.1,1))$.

Suppose now that the trade in Example 5' is authorized by the regulator. Let us frame it, as in Example 5, as the juxtaposition of the two independent trades in Examples 3 and 4 , each one occurring within an autarkic economy. What should the regulator do if the one of these two trades is dropped? The removal of one of the trades (and of the corresponding economy) does not introduce any speculation or betting. The number of different beliefs is lower or unchanged. Hence there is no basis for the regulator to change its mind and ban the remaining trade - the remaining trade should be authorized. We term this property: Independence of Irrelevant Trade (IIT).

In Theorem 1 we show that Pareto domination is the unique regulatory principle satisfying U, MA and IIT. The proof is simple and can be illustrated by using our series of examples. The question could be whether the regulator should authorize the trade in Example 1 between Ann and Bill. If it respects U, MA and IIT, the answer is yes.

To see why, let us introduce yet another way to frame Example 5'.

Example 5": There are four agents: Ann, Bill, Cai and Djal. Ann and Bill trade as described in Example 1 and Cai and Djal trade as described in Example 2.

Consider the trade in Example 1 between Ann and Bill. Let us now introduce Cai and Djal. If the trades were as in Example 3 and Example 4, they would be authorized, in compliance with U. Hence the trade in Example 5 and Example 5' would be authorized, 
in compliance with MA. Since the trade in Example 5' can be decomposed into two unrelated trades in two autarkic economies according to Example 5", the trades in Example 1 and Example 2 would be authorized, in compliance with IIT. Hence a regulator which acts consistently to the three properties U, MA and IIT should authorize the trade in Example 1, as any other Pareto improvement.

\section{Related literature}

In this subsection, we give a broad overview of the literature. We will propose a more detailed and technical discussion of how the literature confronts our axioms after the model and our results are presented.

Stiglitz (1989) discusses taxes on transactions in financial markets to curb trading based on differences in beliefs. In a series of papers Posner \& Weyl (2013, 2013a, 2013b, 2014) advocate that benefit-cost analysis should be used to evaluate the consequences on welfare of new financial products. Though vast amounts of data documenting past trading might be available, it remains a challenge to compute welfare in case of heterogeneous beliefs.

Another strand of the literature has circumvented the challenge by proposing refinements of Pareto domination. Mongin (1997) suggests that the unanimity underpinning Pareto domination can be 'spurious', in the sense that it is based on differences in beliefs and tastes that offset each other. Hence the requirement that unanimity be founded on the same reasoning. Along this line, Gilboa et al. (2014) suggest that a trade should improve the welfare of every agent not only for her own belief but also for some hypothetical common belief. In the same vein, Gayer et al. (2014) strengthen the refinement in Gilboa et al.: A trade should improve the welfare of every agent for everybody's beliefs. Following another route, Brunnermeier et al. (2014) propose a Bergsonian welfare function and suggest that a trade should improve aggregate welfare for everybody's beliefs. The refinements in Gilboa et al. (2014) and in Gayer et al. (2014) both satisfy U and IIT, but violate MA. There are two interpretations of the refinement in Brunnermeier et al. (2014). One satisfies U, but violates MA and IIT, the other violates all three properties.

Blume et al. (2014) use different welfare functions to evaluate different financial market designs. It is found that combinations of restrictions on the set of traded assets, borrowing limits and transaction taxes can offer substantial welfare gains relative to complete financial markets. Blume et al. (2014) and Posner \& Weyl (2013) are both suggesting that financial innovation can destroy welfare.

Duffie (2014) criticizes this literature. First, there is no compelling philosophical justification for banning speculative trade between consenting agents. Second, there is no normative foundation for treating beliefs and tastes different: both are parts of personal preferences. Third, beliefs and tastes cannot be separated: heterogeneous beliefs with state- 
independent tastes and identical beliefs with state-dependent tastes are indistinguishable from an observational point of view.

In the present paper we work under the hypothesis that beliefs and tastes can be separated and explore the possibility of having a regulatory principle banning potentially harmful trades. Since we have an impossibility result, we do not perceive the hypothesis to be a limitation.

\section{The model}

We use pure exchange economies with one good in every state as our framework. Since we solely study changes from one allocation to another allocation there is no need to consider endowments. Generalizations to multi-good economies and production economies easily follow.

\section{The setup}

There is a finite number of states $s \in\{1, \ldots, S\}$. Let $\triangle=\left\{\pi \in \mathbb{R}_{+}^{S} \mid \sum_{s} \pi^{s}=1\right\}$ be the set of probability distributions on the set of states. In every state $s$ there is one good.

A consumer $i$ is described by her consumption set $X_{i} \subset \mathbb{R}$, Bernoulli utility function $u_{i}$ : $X_{i} \rightarrow \mathbb{R}$ and belief $\pi_{i} \in \triangle$. If consumer $i$ plans to consume the bundle $x_{i}=\left(x_{i}^{1}, \ldots, x_{i}^{S}\right) \in X_{i}^{S}$, then her expected utility is $\sum_{s} \pi_{i}^{s} u_{i}\left(x_{i}^{s}\right)$.

An economy $E$ consists of a finite number of consumers so $E=\left(X_{i}, u_{i}, \pi_{i}\right)_{i \in \mathscr{M}}$ where $\mathscr{M}=\{1, \ldots, m\}$. A common-belief economy is an economy $E$ with $\pi_{i}=\pi_{h}$ for every $i, h \in$ $\mathscr{M}$. For a pair of economies $E$ and $E^{\prime}$, the economy $\left(E, E^{\prime}\right)$ is simply the juxtaposition of $E$ and $E^{\prime}$.

Consider an economy $E$. An allocation is an $m$-tuple of consumption plans $x=\left(x_{i}\right)_{i \in \mathscr{M}}$ so $x_{i} \in X_{i}$ for every $i$. For an allocation $x$ a trade is a $m$-tuple $\mu=\left(\mu_{i}\right)_{i \in \mathscr{M}}$ with $\sum_{i} \mu_{i}=0$ such that $x+\mu$ is an allocation so $x_{i}+\mu_{i} \in X_{i}$ for every $i$. Let $\mathscr{M}(\mu)=\left\{i \in \mathscr{M} \mid \mu_{i} \neq 0\right\}$ be the set of consumers $i$ who trade. An improving trade is a trade $\mu$ where for every $i \in \mathscr{M}(\mu)$,

$$
\sum_{s} \pi_{i}^{s} u_{i}\left(x_{i}^{s}+\mu_{i}^{s}\right)>\sum_{s} \pi_{i}^{s} u_{i}\left(x_{i}^{s}\right)
$$

An improvement is an allocation and an improving trade $(x, \mu)$.

Consider an improvement $(x, \mu)$ for economy $E$ and an improvement $\left(x^{\prime}, \mu^{\prime}\right)$ for economy $E^{\prime}$. Then the juxtaposition of the improvements $\left(\left(x, x^{\prime}\right),\left(\mu, \mu^{\prime}\right)\right)$ is an improvement for the juxtaposition of the economies $\left(E, E^{\prime}\right)$.

Improvements are submitted to a regulator which either authorizes or bans them. The regulator knows the characteristics of the agents but has no other information. In particular 
the regulator does not have any information about the likelihood of the different states. The regulator is described by a function that maps economies $E$ and improvements $(x, \mu)$ into recommendations $\{0,1\}$ where $\Gamma(E, x, \mu)=1$ corresponds to authorizing the improvement and $\Gamma(E, x, \mu)=0$ corresponds to banning the improvement.

The upcoming questions are: What could be desirable properties for authorization functions? How do authorization functions having these properties perform?

\section{Three properties of regulation}

Recall that speculative trades are characterized by two properties: agents having different beliefs; and agents trading in order to take advantage of other agents having other beliefs.

A first question naturally arises: What should the policy recommend for common-belief economies and improvements? Since all agents have identical beliefs, trades are, by definition, not speculative. Consequently all such trades should be authorized.

Unanimity (U) For a common-belief economy $E$ and an improvement $(x, \mu)$,

$$
\Gamma(E, x, \mu)=1 .
$$

It seems natural that the regulator should authorize all improvements in case of identical beliefs. It can be seen as a classical unanimity condition: if all consumers have a common belief and strictly prefer $x+\mu$ to $x$, then the improvement should be authorized.

Violating Axiom $U$ would make sense in case the regulator had additional knowledge. However the starting point of the paper is that the regulator does not have any information about the likelihood of the different states.

A second question naturally arises: What should the regulator do when there is actually no trade between agents with different beliefs (and thus no one tries to take advantage of other agents having other beliefs)? Suppose the regulator authorized finitely many independent improvements in finitely many autarkic common-beliefs economies. Should these trades be banned if now submitted to the regulator as one trade - i.e. should the mere juxtaposition of the finitely many original trades result in trades being banned? Since juxtaposing the trades does not introduce any speculation or betting, the regulator should not change its mind. After all, agents solely trade with other agents having the same belief as themselves, hence the trades are not speculative, and should therefore be authorized.

Merge-Proofness of Autarky (MA) For finitely many common-belief economies $E, \ldots, E^{\prime}$ and finitely many improvements $(x, \mu), \ldots,\left(x^{\prime}, \mu^{\prime}\right)$,

$$
\Gamma(E, x, \mu)=\ldots=\Gamma\left(E^{\prime}, x^{\prime}, \mu^{\prime}\right)=1
$$


implies

$$
\Gamma\left(\left(E, \ldots, E^{\prime}\right),\left(x, \ldots, x^{\prime}\right),\left(\mu, \ldots, \mu^{\prime}\right)\right)=1
$$

Axiom MA eliminates some forms of excessive inertia. Consider again the introductory examples, and imagine $N$ replicas of the pair Ann and Cai (Example 3) and one pair of Bill and Djal (Example 4). For a large $N$, the economy is almost showing homogeneous beliefs. For a rule violating MA, the presence of an outlier (here Bill and Djal), not involved in any trade with the rest of the economy, would block the entire economy.

A third question naturally arises: What should the regulator do when part of an economy and an improvement are dropped? Suppose the regulator authorizes the juxtaposition of two independent trades in the juxtaposition of two autarkic economies. Should the trade for one of the economies be banned just because the other trade and the other economy are dropped? Dropping one of the trades (and of the corresponding economy) does not introduce any speculation or betting. The number of different beliefs is lower or unchanged. Hence there is no basis for the regulator to change its mind and ban the remaining trade - the remaining trade should be authorized.

Independence of Irrelevant Trade (IIT) For two economies $E, E^{\prime}$ and two improvements $(x, \mu),\left(x^{\prime}, \mu^{\prime}\right)$,

$$
\Gamma\left(\left(E, E^{\prime}\right),\left(x, x^{\prime}\right),\left(\mu, \mu^{\prime}\right)\right)=1
$$

implies

$$
\Gamma(E, x, \mu)=1 \text {. }
$$

Axiom IIT eliminates some forms of framing. Consider again Example 5' and imagine the trade is authorized. The trade can be seen as Ann and Bill trading as in Example 1 and Cai and Djal trading as in Example 2 or Ann and Cai trading as in Example 3 and Bill and Djal trading as in Example 4. The trade should not be banned just because either Cair and Djal or Bill and Djal are dropped. The two agents left trade just like before the other two agents were dropped.

\section{The main result}

In the present section we first show that our three axioms characterize Pareto domination, discuss how the refinements proposed in the literature fare with respect to our axioms and explain how the analysis extends to ambiguous beliefs. 


\section{Laissez-faire}

Our three axioms characterize laissez-faire.

Theorem 1 An authorization function $\Gamma$ satisfies U, MA and IIT if and only iffor all economies E and all improvements $(x, \mu)$,

$$
\Gamma(E, x, \mu)=1
$$

Proof: We leave it to the reader to check that the authorization function with $\Gamma(E, x, \mu)=1$ for all economies $E$ and improvements $(x, \mu)$ satisfies U, MA and IIT. Consequently we shall focus on the converse claim.

Consider an economy $E$ and an improvement $(x, \mu)$. Define $|\mathscr{M}|$ economies $\left(E_{i}\right)_{i \in \mathscr{M}}$ with consumer $i$ and a new consumer $i+m$ in economy $E_{i}$ for every $i \in \mathscr{M}(\mu)$ and consumer $i$ and two new consumers $i+m$ and $i+2 m$ for every $i \notin \mathscr{M}(\mu)$.

For $i$ with $\mu_{i} \in \mathbb{R}^{S} \backslash \mathbb{R}_{+}^{S}$ suppose the new consumer $i+m$ in economy $E_{i}$ has elementary utility function $u_{i+m}\left(x_{i+m}^{s}\right)=\ln \left(x_{i+m}^{s}\right)$ and beliefs $\pi_{i+m}=\pi_{i}$. Let the consumption plan $x_{i+m}$ for consumer $i+m$ be defined by

$$
x_{i+m}^{s}=\left\{\begin{array}{cc}
\varepsilon & \text { for } \mu_{i}^{s}<0 \\
1+\mu_{i}^{s} & \text { for } \mu_{i}^{s} \geq 0
\end{array}\right.
$$

where $\varepsilon>0$. Since $\lim _{\varepsilon \rightarrow 0} U_{i+m}\left(x_{i+m}\right)=-\infty$ and $\lim _{\varepsilon \rightarrow 0} U_{i+m}\left(x_{i+m}-\mu_{i}\right)>-\infty$ there is $\varepsilon>0$ such that $U_{i+m}\left(x_{i+m}\right)<U_{i+m}\left(x_{i+m}-\mu_{i}\right)$. Therefore $z_{i}=\left(\left(x_{i}, x_{i+m}\right),\left(\mu_{i},-\mu_{i}\right)\right)$ is an improvement for the common-belief economy $E_{i}$ for every $i \in \mathscr{M}(\mu)$.

For $i$ with $\mu_{i} \notin \mathbb{R}^{S} \backslash \mathbb{R}_{+}^{S}$ suppose the new consumers $i+m$ and $i+2 m$ has elementary utility function $u_{k}\left(x_{k}^{s}\right)=\ln \left(x_{k}^{s}\right)$ and beliefs $\pi_{k}=\pi_{i}$ for $k \in\{i+m, i+2 m\}$. Consider an arbitrary vector $v \in \mathbb{R}^{S}$ such that $0.5 \mu_{i}+v \in \mathbb{R}^{S} \backslash \mathbb{R}_{+}^{S}$, let $x_{i+m}$ and $x_{i+2 m}$ be defined by

$$
\begin{gathered}
x_{i+m}^{s}=\left\{\begin{array}{cc}
\varepsilon & \text { for } 0.5 \mu_{i}^{s}-v^{s}<0 \\
1-\left(0.5 \mu_{i}^{s}+v^{s}\right) & \text { for } 0.5 \mu_{i}^{s}-v^{s} \geq 0
\end{array}\right. \\
x_{i+2 m}^{s}=\left\{\begin{array}{cc}
\varepsilon & \text { for } 0.5 \mu_{i}^{s}+v^{s}<0 \\
1+\left(0.5 \mu_{i}^{s}+v^{s}\right) & \text { for } 0.5 \mu_{i}^{s}+v^{s} \geq 0
\end{array}\right.
\end{gathered}
$$

where $\varepsilon>0$. Let $\mu_{i+m}=-0.5 \mu_{i}+v$ and $\mu_{i+2 m}=-0.5 \mu_{i}-v$. Since $\lim _{\mathcal{E} \rightarrow 0} U_{k}\left(x_{k}\right)=-\infty$ and $\lim _{\varepsilon \rightarrow 0} U_{k}\left(x_{k}+\mu_{k}\right)>-\infty$ for $k \in\{i+m, i+2 m\}$ there is $\varepsilon>0$ such that $U_{k}\left(x_{k}\right)<$ $U_{k}\left(x_{k}+\mu_{k}\right)$ for $k \in\{i+m, i+2 m\}$. Therefore $z_{i}=\left(\left(x_{i}, x_{i+m}, x_{i+2 m}\right),\left(0, \mu_{i},-\mu_{i}\right)\right)$ is an improvement for the common-belief economy $E_{i}$ for every $i \notin \mathscr{M}(\mu)$.

According to $\mathrm{U}, \Gamma\left(E_{i}, z_{i}\right)=1$ for every $i$ because $E_{i}$ is a common-belief economy for every $i$. According to MA, 


$$
\Gamma\left(\left(E_{i}\right)_{i},\left(z_{i}\right)_{i}\right)=1
$$

According to IIT,

$$
\Gamma(E, x, \mu)=1 .
$$

Hence laissez-faire is the unique policy satisfying U, MA and IIT.

Our theorem is disturbing for anyone sympathetic to the general agenda of protecting consumers against the consequences of trade based on distorted beliefs. Probably even more disturbing is the fact that restricting improvements to be equilibrium allocations associated with Walrasian equilibria does not change Theorem 1 as we show in Corollary 1.

Corollary 1 Suppose that for all economies $E$ and all improvements $(x, \mu)$ there are prices $p \in \mathbb{R}_{++}^{S}$ such that for every $i, x_{i}+\mu_{i}$ is a solution to

$$
\begin{array}{cl}
\max _{c_{i}} & u_{i}\left(c_{i}\right) \\
\text { s.t. } & p \cdot c_{i}=p \cdot x_{i} .
\end{array}
$$

Then an authorization function $\Gamma$ satisfies $U, M A$ and IIT if and only if

$$
\Gamma(E, x, \mu)=1 .
$$

Proof: The proof follows the proof of Theorem 1 except the choices of $x_{i+m}$ in case $\mu_{i} \neq 0$ and $x_{i+m}$ and $x_{i+2 m}$ as well as $\mu_{i+m}$ and $\mu_{i+2 m}$ in case $\mu_{i}=0$ are more delicate.

In case $\mu_{i} \in \mathbb{R}^{S} \backslash \mathbb{R}_{+}^{S}, x_{i+m}$ has to be chosen such that $x_{i+m} \in \mathbb{R}_{++}^{S}$ and $x_{i+m}-\mu_{i}$ is the solution to the consumer problem for consumer $i+m$. In case $\mu_{i}=0$, first $v \in \mathbb{R}^{S}$ has to be chosen such that $v \neq 0$ and $p \cdot v=0$, second $x_{i+m}$ and $x_{i+2 m}$ has to be chosen such that $x_{i+m}, x_{i+2 m} \in \mathbb{R}_{++}^{S}$ and $x_{i+m}+v$ is the solution to the consumer problem for consumer $i+m$ and $x_{i+2 m}-v$ is the solution to the consumer problem for consumer $i+2 m$. Both cases are trivial.

A way to escape the implication of our theorem and its corollary could be to regulate trades one-by-one. In the appendix we show that regulation of trades one-by-one can be subject to framing effects and introduce a property eliminating some of the framing effects. Again the result is that laissez-faire is the unique rule satisfying our four properties.

\section{Refinements of Pareto domination}

Gilboa et al. (2014) propose a criterion to separate improvements triggered by differences in beliefs as in Example 1 from improvements triggered by differences in insurance needs as in Examples 2, 3 and 4. They require that there is some hypothetical common belief such that 
the improvement remains an improvement for this common belief. The resulting refinement of Pareto domination is denoted No-Betting Pareto (NBP) dominance.

Regulation based on NBP dominance would satisfy U and IIT, but violate MA. Indeed, applied to the introductory series of examples, an NBP-compliant regulator would authorize the trades in Examples 2, 3 and 4, but ban the trades in 1 and 5. The reason why a NBPcompliant regulator would ban the trade in Example 5 is that there is no common belief under which both Ann and Bill would be better off. The problem is neither that they trade with each other nor that they bet on their trading partner having mistaken beliefs as in Example 1: Both trade with someone having the same belief as they themselves have. The problem is that their independent and unrelated actions cannot be rationalized by a common belief. And this lack of rationalization could lead to reservations about whether the improvement in Example 5 is actually an improvement.

Another criterion is proposed in Gayer et al. (2014). They require that for the belief of every agent the improvement remains an improvement for this belief as common hypothetical belief. Clearly the resulting refinement denoted Unanimity Pareto dominance is a strengthening of Gilboa et al. (2014), but nevertheless it satisfies U and IIT, but violates MA as illustrated by examples 3, 4 and 5 .

Brunnermeier et al. (2014) take some set of beliefs as being 'reasonable' and require that an improvement has to improve social welfare for all beliefs in the set of reasonable beliefs as common hypothethical beliefs. The resulting refinement of Pareto domination is denoted Belief-Neutral Social Welfare. In case the set of reasonable beliefs is a subset of the convex hull of the beliefs of the agents the criterion would satisfy U, but violate MA and IIT. In case the set of reasonable beliefs is unrelated to the beliefs of the agents the criterion would violate $\mathrm{U}$ in addition to MA and IIT.

For a violation of MA consider two economies with two consumers and four states. In the first economy, let $e_{i} \in \mathbb{R}^{4}$ be the excess utility across states of consumer $i \in\{A, B\}$ with $e_{A}=(2,-1,1,-3)$ and $e_{B}=(-1,2,-3,1)$. Suppose the two consumers have identical beliefs $\pi_{1}$ with $\pi_{1}^{1}=\pi_{1}^{2}>2 \pi_{1}^{3}=2 \pi_{1}^{4}$. Then $\pi_{1} \cdot e_{i}>0$ for both $i$ in the first economy. In the second economy, let $e_{C}=(1,-3,2,-1)$ for consumer $C$ and $e_{D}=(-3,1,-1,2)$ for consumer $D$. Suppose the two consumers have identical beliefs $\pi_{2}$ with $\pi_{2}^{1}=\pi_{2}^{2}<0.5 \pi_{2}^{3}=$ $0.5 \pi_{2}^{4}$. Then $\pi_{2} \cdot e_{i}>0$ for both $i$ in the second economy. Suppose the set of reasonable beliefs for every economy is the convex hull of the set of beliefs of the consumers in the economy and the regulator uses the utilitarian welfare function. Then the regulator would authorize the trades in the two economies, but ban the trades in the juxtaposed economy because $e_{A}+e_{B}+e_{C}+e_{D}=(-1,-1,-1,-1)$.

For a violation of IIT consider an economy with four consumers and four states. Suppose the first two consumers $i \in\{A, B\}$ trade with each other and last two consumers 
$i \in\{C, D\}$ trade with each other. For excess utilities and beliefs for the consumers let $e_{A}=(1,-2,1,-2)$ and $\pi_{A}^{1}=\pi_{A}^{3}>2 \pi_{A}^{2}=2 \pi_{A}^{4}, e_{B}=(-2,1,-2,1)$ and $\pi_{B}^{1}=\pi_{B}^{3}<0.5 \pi_{B}^{2}=$ $0.5 \pi_{B}^{4}, e_{C}=(-1,3,-1,3)$ and $\pi_{C}^{1}=\pi_{C}^{3}<3 \pi_{C}^{2}=3 \pi_{C}^{4}$ and $e_{D}=(3,-1,3,-1)$ and $\pi_{D}^{1}=$ $\pi_{D}^{3}>(1 / 3) \pi_{D}^{2}=(1 / 3) \pi_{D}^{4}$. Suppose the set of reasonable beliefs for every economy is the convex hull of the set of beliefs of the consumers in the economy and the regulator uses the utilitarian welfare function. Then the regulator would authorize the trades because $e_{A}+e_{B}+e_{C}+e_{D}=(1,1,1,1)$, but ban the trade in the economy with consumers $A$ and $B$ because $e_{A}+e_{B}=(-1,-1,-1,-1)$.

For a violation of $U$ in case the set of reasonable beliefs is fixed. Consider the consumers $A$ and $B$ in the example of a violation of MA. Suppose the set of reasonable beliefs for every economy is a singleton $\pi$ with $\pi^{1}=\pi^{2}<2 \pi^{3}=2 \pi^{4}$ and the regulator uses the utilitarian welfare function. Then it would ban the trade.

The contentious axiom for the literature is MA according to which the mere presence of agents with different beliefs in the same state space does not warrant coercive public action. Indeed, as long as agents trade with other agents with identical beliefs, trade should be authorized. We could try to argue for the opposite. Assume two agents can both take a pink pill or not where one of the actions is lifesaving and the other is lethal for both agents. Suppose the two agents have opposite beliefs about consequences of the two actions. If one of the agents comes to the regulator and exposes its belief and plan, then the regulator can say nothing else than: 'try your chosen action, and we'll see whether you'll die or not'. But if both agents come to the regulator with their opposite beliefs, then the regulator knows for sure that one of the agents is going to die and is facing a troubling conflict.

Forgetting about what the alternative to no-action would be in such a situation, it is reminiscent of Pascal's wager: We all live with mutually exclusive states, either God exists, or He does not. For any non-trivial probability that God exists, one wants to be observant of religious rules given the pleasure of going to heaven and/or the pain of not going. Nevertheless some of us are not observant. And there is no probability of God's existence that rationalizes both observance and non-observance. Consequently our sense is that the reservations about whether the trade in Example 5 is actually an improvement build on considerations that are very pervasive. We argue that opening the way to coercion based on the fact that people live with the same state space could drive public action to intrusive shores.

\section{Ambiguous beliefs}

Our analysis and results straightforwardly extend to the case of ambiguous beliefs where consumers have sets of beliefs $\Pi_{i} \subset \triangle$, not necessarily singletons. Preferences can be as in, e.g., Bewley (2002) or Gilboa \& Schmeidler (1989) according to which consumer $i$ prefers bundle $x_{i}$ to bundle $x_{i}^{\prime}$ provided respectively $\sum_{s} \pi_{i}^{s} u_{i}\left(x_{i}^{s}\right) \geq \sum_{s} \pi_{i}^{s} u_{i}\left(x_{i}^{s}\right)$ for all $\pi_{i} \in \Pi_{i}$ or 
$\min _{\pi_{i} \in \Pi_{i}} \sum_{s} \pi_{i}^{s} u_{i}\left(x_{i}^{s}\right) \geq \min _{\pi_{i} \in \Pi_{i}} \sum_{s} \pi_{i}^{s} u_{i}\left(x_{i}^{\prime s}\right)$.

A common-belief economy in case of ambiguity is an economy $E$ where consumers have identical sets of beliefs: $\Pi_{i}=\Pi_{h}$ for every $i, h \in \mathscr{M}$. Given this extended definition of common-belief economies, all our axioms readily extend. It could be argued that coincidence of sets of beliefs is less likely than coincidence of singleton beliefs (if, e.g., beliefs are taken from a finite set, as percentages); in which case our axioms become even weaker. Finally it can be checked immediately that our results remain valid.

\section{References}

Bewley, T.: Knightian decision theory. Part I, Decisions in Economics and Finance 25, 79-110 (2002).

Blume, L., Cogley, T., Easley, D., Sargent, T., Tsyrennikov, V.: The Case for Incomplete Markets, working paper (2014).

Brunnermeier, M. K., Simsek, A., Xiong, W.: A Welfare Criterion for Models with Distorted Beliefs, Quarterly Journal of Economics 129, 1711-1752 (2014).

Duffie, D.: Challenges to a Policy Treatment of Speculative Trading Motivated by Differences in Beliefs, Journal of Legal Studies 43, 173-182 (2014).

Gayer, G., Gilboa, I., Samuelson, L., Schmeidler, D.: Pareto Efficiency with Different Beliefs, Journal of Legal Studies 43, 151-172 (2014).

Gilboa, I., Samet, D., Schmeidler, D.: Utilitarian Aggregation of Beliefs and Tastes, Journal of Political Economy 112, 932-938 (2004).

Gilboa, I., Samuelson, L., Schmeidler, D.: No-Betting-Pareto Dominance, Econometrica 82, 1405-1442 (2014).

Gilboa, I., Schmeidler, D.: Maxmin utility with non-unique prior, Journal of Mathematical Economics 18, 141-153 (1989).

Mongin, P.: Spurious Unanimity and the Pareto Principle, working paper (1997).

Posner, E., Weyl, G.: Benefit-Cost Analysis for Financial Regulation, American Economic Review 103, 1-5 (2013).

Posner, E., Weyl, G.: An FDA for Financial Innovation: Applying Insurable Interest Doctrine to 21st Century Financial Markets, Northwestern University Law Review 107, 1307-1357 (2013a). 
Posner, E., Weyl, G.: Benefit-Cost Paradigms in Financial Regulation, working paper (2013b).

Posner, E., Weyl, G.: Benefit-Cost Analysis for Financial Regulation: A Response to Criticisms, working paper (2014).

Stiglitz, J.: Using tax policy to curb speculative trading, Journal of Financial Services Research 3, 101-115 (1989).

\section{Appendix}

A way to circumvent that beliefs in unrelated trades can block trades is to limit the regulator to consider trades one-by-one. Unfortunately such a regulatory principle is subject to framing effects. We show that in order to avoid framing effects the regulatory rule has to be laissez-faire.

\section{Multiple trades}

Examples 5 and 5' involve two different packages of trades, but in both cases the four agents start with the same initial allocation $((1,1),(1,1),(2,0.1),(0.1,2))$ and end up with the same final allocation $((2,0),(0,2),(1,1.1),(1.1,1))$. Regulation based on the different refinements of Pareto optimality would ban trade between Ann and Bill in Example 5', while the trade between Cai and Djal would be authorized. Similarly the trades between Ann and Cai as well as between Bill and Djal in Example 5 would be authorized.

There is no fundamental economic reason to differentiate between the two packages. They start from exactly the same initial allocation, end up at exactly the same final allocation and involve exactly the same beliefs. The sole difference is in the accounting of the clearing house. The accounting is virtual and arbitrary, consequently we suggest that it should not matter. Hence the regulator should not differentiate between these packages of trades. Otherwise, it would be subject to a framing effect, pushing agents to waste time looking for trading partners who enable them to circumvent the regulatory principle.

In order to avoid framing effects in regulation of trades one-by-one we propose a fourth limit on regulation. It requires that the evaluation of every trade should depend on neither the pattern of trade resulting in the allocation reached before the trade nor the pattern of trade after the trade. In Corollary 2 we consider finite lists of trades and show that the Pareto principle is the unique principle satisfying U, MA and IIT for one trade and Pattern Independence for lists of trades. 


\section{No framing}

In order to tackle framing effects arising from packaging the same trade in different ways, we need a richer setting where initial allocations and lists of trades rather than one trade are evaluated. In such a setting the regulator can distinguish between different packages of trades starting from the same initial allocation and ending at the same final allocation.

Now an improvement is a feasible allocation and a finite list of $n$ trades $\left(x,\left(\mu_{a}\right)_{a}\right)$ such that every trade $\mu_{c}$ is an improving trade of the allocation obtained by starting with the feasible allocation and adding the previous trades to it $x+\sum_{b<c} \mu_{b}$. A one-trade improvement is an improvement with one trade $(x, \mu)$.

With the change from one-trade improvements to multi-trade improvements the authorization function changes to a function from economies and multi-trade improvements $\left(E, x,\left(\mu_{a}\right)_{a}\right)$ to multi-authorizations of trades $\{0,1\}^{n}$. Banning a trade can change a subsequent trade from being improving to not being improving. However no assumption needs to be made about evaluation of non-improving trades.

Our three properties U, MA and IIT carry over to the setting with lists of trades provided they are restricted to one-trade improvements. In addition to these three properties, we propose that regulation of a trade should depend on the intermediate allocations from which it starts and to which it ends and nothing else. Hence, regulation of a trade should be independent of the patterns of trade before and after it.

Pattern Independence (PI) For an economy $E$ and an improvement $\left(x,\left(\mu_{a}\right)_{a}\right)$,

$$
\Gamma_{b}\left(E, x,\left(\mu_{a}\right)_{a}\right)=1
$$

for every $b<c$ implies

$$
\Gamma_{c}\left(E, x,\left(\mu_{a}\right)_{a}\right)=\Gamma\left(E, x+\sum_{b<c} \mu_{b}, \mu_{c}\right) .
$$

The PI axiom does not eliminate framing effects, but it implies that regulation of lists of trades should be trade by trade. Moreover, PI is sufficient to pin down Pareto domination in a setting with lists of trades.

Corollary 2 An authorization function $\Gamma$ satisfies $U$, MA and IIT for all one-trade improvements and PI for all improvements if and only if for all economies $E$ and all improvements $\left(x,\left(\mu_{a}\right)_{a}\right)$,

$$
\Gamma\left(E, x,\left(\mu_{a}\right)_{a}\right)=(1, \ldots, 1) .
$$

Proof: We leave it to the reader to check that the authorization function with

$$
\Gamma\left(E, x,\left(\mu_{a}\right)_{a}\right)=(1, \ldots, 1)
$$


for all $\left(E, x,\left(\mu_{a}\right)_{a}\right)$ satisfies U, MA, IIT and PI as specified in Corollary 2. Consequently we shall focus on the converse claim.

Consider an economy $E$ and an improvement $\left(x,\left(\mu_{a}\right)_{a}\right)$. According to U, MA and IIT as shown in Theorem 1 , for every $c, \Gamma_{c}\left(E, x+\sum_{b<c} \mu_{b}, \mu_{c}\right)=1$. Then, according to PI, $\Gamma_{c}\left(E, x,\left(\mu_{a}\right)_{a}\right)=1$ for every $c$.

Corollary 2 is reminiscent of Proposition 3 in Gayer et al. (2014) which states that if the set of expected utility profiles is rectangular and convex, then the transitive closure of the preference relation defined by No-Betting-Pareto dominance is the standard Pareto domination. However it must be noted that typically economies do not satisfy rectangularity. 